\title{
Poincaré-Invariant Structures in the Solution Manifold of a Nonlinear Wave Equation
}

Irving $\mathrm{E}$. Segal

Dedicated to Alberto P. Calderón

\begin{abstract}
The solution manifold $\mathfrak{T}$ of the equation $\square \phi+g \phi^{3}=0$ in Minkowski space is studied from the standpoint of the establishment of differential-geometric structures therein. It is shown that there is an almost Kähler structure globally defined on $\mathfrak{M}$ that is Poincaré invariant. In the vanishing curvature case $g=0$ the structure obtained coincides with the complex Hilbert structure in the solution manifold of the real wave equation. The proofs are based on the transfer of the equation to an ambient universal space-time.
\end{abstract}

\section{Introduction}

The solution manifolds of nonlinear wave equations such as $\square \phi+g \phi^{3}=0$ come equipped with natural invariant symplectic structures, in terms of which they appear as infinite-dimensional Hamiltonian systems (cf. [8] and references given there). In connection with quantum field theory and general mathematical questions, Riemannian, Kähler, and measure-theoretic structures in the solution manifold have been of interest. These are well known in 
the linear case, and already in this case it is clear that these structures are inherently global in space-time, unlike the symplectic structure, which even in the nonlinear case can be established locally, quite explicitly in fact. This global feature involves the temporal asymptotics and thereby scattering theory, which was used to establish an almost Kähler structure in the solution manifold near the zero solution for similar equations.

The requisite analysis involves connections with stability theory (cf. [4]), and while the resulting structures are in principle explicit, their expression is relatively complicated, in addition to being only local on the solution manifold. The transfer of a conformally invariant wave equation from Minkowski space $\mathfrak{T}_{0}$ to the universal cosmos $\tilde{\mathfrak{T}} \mathrm{T}$ has proved to be a powerful method for its global treatment (cf. [3], [6], [7]). This method is here used to treat the simplest nontrivial nonlinear such equation and derive an essentially very simple Riemannian structure in the solution manifold and related elements of structure.

\section{The Banach and Symplectic Manifold Structures}

These structures are much more simply and invariantly treated in $\tilde{\mathfrak{T}} \mathrm{K}$ than in $\mathfrak{T}_{0}$. The natural, or «Einstein» energy in $\tilde{\mathfrak{T}}$, is slightly larger than the natural, «Minkowski» energy in $\mathfrak{T}_{0}$, and in practice the limitation to solutions of finite Einstein energy is an inessential constraint. Thus Cauchy data for the cited equation will have finite Einstein energy if they have finite Minkowski energy and if in addition their product with the euclidean distance is such. All such solutions in $\mathfrak{N T}_{0}$ (having Cauchy data of finite Einstein energy at one time) extend canonically and uniquely to $\tilde{\mathfrak{T}}$, in the sense that there exists a unique solution $\tilde{\phi}$ of the equation

$$
\square^{\prime} \tilde{\phi}+g \tilde{\phi}^{3}=0
$$

on $\tilde{\mathfrak{T}}$, of finite Einstein energy

$$
E=\int_{S^{3}}\left[(\operatorname{grad} \tilde{\phi})^{2}+\dot{\tilde{\phi}}^{2}+(1 / 4) \tilde{\phi}^{4}\right] d_{3} V,
$$

such that $\phi=p \tilde{\phi} \mid \mathscr{T T}_{0}$, where $p$ is a fixed smooth function on $\tilde{\mathfrak{T}}$, independent of $\tilde{\phi}$, and $\square^{\prime}$ is the operator in $\tilde{T} \mathcal{T}$ that is covariantly related to the usual D'Alembertian $\square$ in $\mathfrak{T l}_{0}$. For explicit expressions, cf. loc. cit. In more invariant terms, $\tilde{\phi}$ is a section of the scalar bundle of conformal weight 1 , and if smooth is transformed by a conformal transformation $g$ on $\tilde{\mathfrak{N}}$ into another solution of equation $\left({ }^{*}\right)$. The universal cover of the group of all conformal transformations on $\tilde{T} \mathcal{T}$ will be denoted as $\tilde{G}$; more exactly, $\tilde{G}$ will denote the connected component of this group, which is locally isomorphic to $\mathrm{SU}(2,2)$. 
The main presently relevant features are summarized in

Theorem 1. The manifold of all finite Einstein energy solutions of equation $\left(^{*}\right)$ is a Banach manifold relative to the Einstein energy norm in the tangent spaces, representable by the solution manifolds of the first-order variational equations

$$
\square^{\prime} \eta+3 g \tilde{\phi}^{2} \eta=0 .
$$

The conformal group G leaves this manifold invariant, acts smoothly on it, and leaves invariant the symplectic structure defined by the equation

$$
\Omega_{\tilde{\phi}}\left(\eta, \eta^{\prime}\right)=\int_{S^{3}}\left(\dot{\eta} \eta^{\prime}-\eta \dot{\eta}^{\prime}\right) d_{3} U
$$

(the latter integral being independent of the fixed Einstein time involved in the integration).

The proof is part contained in and in part derives straightforwardly from the given references.

\section{The Riemannian Structure}

I first recall the «flat» Riemannian structure in the «free» case in which $g=0$. If $\eta_{i}(i=1,2)$ are two solutions of the wave equation on $\tilde{\mathfrak{T}}$, i.e. distributions satisfying the equation $\square^{\prime} \eta_{i}=0$, there is a canonical inner product applicable to sufficiently regular solutions: $\left\langle\eta_{1}, \eta_{2}\right\rangle$, which is invariant under the group $\tilde{G}$, which acts on solutions as sections of the scalar bundle of weight 1 ; of [5]. The precise expressions for this inner product will not be required explicitly, but the following expression indicates the exact regularity requirement. Let $C$ denote $\left(1-\Delta_{c}\right)^{1 / 4}$, where $\Delta_{c}$ denotes the Laplace-Beltrami operator on $S^{3}$. Then $\langle\eta, \eta\rangle=\left\|C \eta\left(t_{0}, \bullet\right)\right\|_{2}^{2}+\left\|C^{-1} \dot{\eta}\left(t_{0}, \bullet\right)\right\|_{2}^{2}$, where the time $t_{0}$ is arbitrary, $\|\bullet\|_{2}$ indicates the norm in $L_{2}\left(S^{3}\right)$, and $\dot{\eta}=(\partial / \partial t) \eta$.

I recall also (cf. [7]) that the boundary of $\mathfrak{T}_{0}$ as imbedded in $\tilde{\mathfrak{T}}$ consists of two cones $C_{ \pm}$, the respective limits as $x_{0}^{\prime} \rightarrow \pm \infty$ of the space-like surfaces $x_{0}=x_{0}^{\prime}$, where $x_{0}$ denotes the Minkowski time (in an arbitrary Lorentz frame). These cones are conformally equivalent to ordinary light-cones in $M_{0}$, apart from their inclusion of a point at spatial infinity, essentially the antipode of the chosen origin in $S^{3}$, which constitutes their intersection.

Theorem 2. Let $M$ denote the manifold of all infinitely differentiable solutions of equation $\left(^{*}\right)$. For any solution $\phi \in M$. Let $G_{\phi}$ denote the following real 
symmetric form on the tangent space $T_{\phi}$ at $\phi:$

$$
G_{\phi}\left(\eta, \eta^{\prime}\right)=\left\langle\eta_{-\infty}, \eta_{-\infty}^{\prime}\right\rangle+3 g \int_{\bar{M}} \phi^{2} \eta \eta^{\prime} d_{4} U,
$$

where $\eta$ and $\eta^{\prime}$ are $C^{\infty}$ tangent vectors and

(1) $\eta_{-\infty}$ denotes the solution of the free wave equation in $\tilde{M}$ having the same restriction to $C_{-}$as $\eta$;

(2) $d_{4} U$ denotes the invariant volume element on $S^{3}$, multiplied by $d t$, $t=$ Einstein time.

Then $G_{\phi}$ is a Riemannian structure on $M$ that is invariant under the action of the Poincaré group, including scaling.

Proof. The restriction to $C_{-}$of $\eta$ provides $C^{\infty}$ Goursat data for the free wave equation, for which the solution is given in [1], showing its existence and regularity. Under a transformation $T$ in the (scaling-inclusive) Poincaré group $\tilde{P}, \phi$ is carried into a solution $T \phi$, and the differential of the transformation on $\phi$ carries $\eta$ into another tangent vector $T \eta$ to $M$. The cone $C_{-}$is invariant under $\tilde{P}$, and $(T \eta)_{-\infty}$ is simply the transform of $\eta_{-\infty}$ under the action of $T$, since this is true of their respective Goursat data. Thus the form $\left\langle\eta_{-\infty}, \eta_{-\infty}^{\prime}\right\rangle$ on $T_{\phi}$ is $\tilde{P}$-invariant, under the action induced from the action of $\tilde{P}$ on the weight 1 scalar bundle.

The integral $\int_{\bar{M}} \phi^{2} \eta \eta^{\prime} d_{4} U$ is unchanged by Poincaré transformation, as shown in [5] (which deals with integrals of the form $\int \phi^{4} d_{4} U$ where $\phi$ is an arbitrary section of the same bundle, from which by polarization the presently relevant invariance follows). Thus the entire form $G_{\phi}$ is Poincaré invariant.

In order to express the results most simply in terms of classical solutions, only $C^{\infty}$ solutions were considered in Theorem 2 , but the result is readily extended to the full Banach manifold, inclusive of generalized solutions, by completion.

Corollary 2.1. Let $M^{\prime}$ denote the manifold of all finite-energy solutions of equation (*). For any solution $\phi \in M^{\prime}$, let $G_{\phi}^{\prime}$ denote the following real symmetric form on the (full) tangent space $T_{\phi}^{\prime}$ to $M^{\prime}$ at $\phi$ :

$$
G_{\phi}^{\prime}\left(\eta, \eta^{\prime}\right)=\left\langle\eta_{-\infty}, \eta_{-\infty}^{\prime}\right\rangle+3 g \int_{\bar{M}} \phi^{2} \eta \eta^{\prime} d_{4} U,
$$

where $\eta$ and $\eta^{\prime}$ are tangent vectors at $\phi$, and $\eta_{-\infty}$ is the solution of the wave equation obtained by completion from the mapping $\eta \rightarrow \eta_{-\infty}$ defined on $C^{\infty}$ solutions, as a map from tangent vector in the finite-energy topology to free solutions in their conformally-invariant norm. Then the analogous conclusion to that of Theorem a holds. 
Proof. The differential equation for the tangent vector guarantees that their Cauchy data remain in the finite-energy space earlier indicated and that temporal evolution is continuous in this norm. Combining results in [2] on the regularity of restrictions to submanifolds of functions of specified regularity with the explicit expression [1] for the conformally invariant norm of free solutions in terms of their Goursat data on $C_{-}$, it follows that the map $\eta \rightarrow \eta_{-\infty}$ is continuous from tangent vectors in the energy norm to free solutions in the conformally invariant norm. Accordingly the map extends by continuity from the dense subset of $C^{\infty}$ solutions of the tangent equation to all those of finiteenergy Cauchy data.

By conservation of energy in the case of $\phi$, and its boundedness for $|t| \leqslant \pi$ in the case of $\eta, \phi$ and $\eta$ are in $L_{6}\left(S^{3}\right)$ at each fixed time (having $L_{2}$ gradients in $S^{3}$ ), with $L_{6}$ norms bounded as a function of $t$. The same is true of their norms in $L_{2}\left(S^{3}\right)$, so that $\int_{\bar{M}} \phi^{2} \eta \eta^{\prime} d_{4} U$ depends continuously on $\phi, \eta$, and $\eta^{\prime}$ in the energy norms appropriate to the Banach manifold under consideration. Thus the entire metric $G_{\phi}\left(\eta, \eta^{\prime}\right)$ extends uniquely and continuously from the case of smooth $\phi, \eta$, and $\eta^{\prime}$, to a metric $G_{\phi}^{\prime}\left(\eta, \eta^{\prime}\right)$ defined for arbitrary $\phi \in M^{\prime}$ and arbitrary tangent vectors $\eta$ and $\eta^{\prime}$ at $\phi$.

\section{Discussion}

The conjunction of the Riemannian with the symplectic structure gives rise canonically to an associated almost complex structure, which is symplectic and positive, thereby giving rise to an almost Kähler structure with modified Riemannian structure but identical symplectic structure. Thus the symplectic structure $\Omega$ on $M^{\prime}$ can be extended to a Poincaré-invariant almost Kähler structure. For the free wave equation this is the linear structure, constituting a complex Hilbert space, left invariant by a known positive-energy unitary representation of $\tilde{G}$. In the nonlinear case it is not known whether the almost Kähler structure established here is infinitesimally integrable.

The Poincaré invariance of the map from Goursat data on $C_{\text {- }}$ to solutions of the nonlinear equation provides a Poincaré-invariant map from measures on the Goursat data space to measures on the solution manifold $M^{\prime}$. Suitable Gaussian measures will thereby be mapped into non-Gaussian measures enjoying non-trivial, invariance and quasi-invariance properties, which may be interpreted as generalized stochastic solutions of the nonlinear equation.

In the language of theoretical physics, the integral expression involved in $G_{\phi}$ may be correlated with the Hessian of the interaction Lagrangian, $(1 / 4) g \int_{\bar{M}} \phi^{4} d_{4} U$. 


\section{References}

[1] Baez, J. C. Conformally invariant quantum fields. Ph. D. Dissertation, Department of Mathematics, M.I.T., June, 1986.

[2] Calderón, A.P. Lebesgue spaces of differentiable functions and distributions. Proc. Symp. Pure Math. IV, 33-49 (1961). Amer. Math. Soc., Providence.

[3] Choquet-Bruhat, Y., Paneitz, S. M., and Segal, I. E. The Yang-Mills equations on the universal cosmos. Jour. Funct. Anal. 53 (1983), 112-150.

[4] Paneitz, S. M. Essential unitarization of symplectics and applications to field quantization. Jour. Funct. Anal. 48 (1982), 310-359.

[5] Paneitz, S. M. and Segal, I. E. Analysis in space-time bundles, I: General considerations and the scalar bundle. Jour. Funct. Anal. 47 (1982), 78-142, II, ibid. 49 (1982), 335-413.

[6] Segal I. E. Induced bundles and nonlinear wave equations. Proc. Conf. honor of G. W. Mackey, Math. Sci. Res. Inst., Berkeley, CA, May, 1984, in press.

[7] Segal, I.E. Reduction of scattering to an invariant finite displacement in an ambient space-time. Proc. Natl. Acad. Sci. USA 81 (1984), 7266-7268.

[8] Segal, I. E. Symplectic structures and the quantization problem for wave equations. Symposia Mathematica XIV (1974), Prof. Conf. Rome 1973.

Irving E. Segal

Massachusetts Institute of Technology

Cambridge, MA 02139

USA

Research supported in part by the NSF. 